\title{
Investigations of the Tritium Recycling in TFTR using the DT Neutron Rate
}

\author{
S.E. Kruger, ${ }^{\dagger}$ R.V. Budny, J.D. Callen, $^{\dagger}$ Z. Chang, C.H. SkInNer, \\ J.D. Strachan, Princeton University, ${ }^{\dagger}$ University of Wisconsin
}

\begin{abstract}
During deuterium-only neutral-beam-injected discharges, tritium from earlier deuterium-tritium discharges is released from the vessel limiters and walls to cause a deuterium-tritium neutron count rate comparable to the deuterium-deuterium neutron count rate. A measure of the tritium density in the plasma based on neutron rate measurements is defined and used to determine which parameters influence tritium influx to the plasma core. The tritium density is observed to decrease in a sequence of deuterium-only supershots and to depend on the amount of tritium injected in prior DT shots and the amount of tritium present in the limiter. A weak correlation is also observed with the plasma current, but not with beam power, hydrogen influx, carbon influx, visible bremsstrahlung, lithium pellet injection, blooms, nor disruptions.
\end{abstract}

\section{DISCLAIMER}

\begin{abstract}
This report was prepared as an account of work sponsored by an agency of the United States Government. Neither the United States Government nor any agency thereof, nor any of their employees, makes any warranty, express or implied, or assumes any legal liability or responsibility for the accuracy, completeness, or usefulness of any information, apparatus, product, or process disclosed, or represents that its use would not infringe privately owned rights. Reference herein to any specific commercial product, process, or service by trade name, trademark, manufacturer, or otherwise does not necessarily constitute or imply its endorsement, recommendation, or favoring by the United States Government or any agency thereof. The views and opinions of authors expressed herein do not necessarily state or reflect those of the United States Government or any agency thereof.
\end{abstract}




\section{Introduction}

During a plasma discharge in TFTR, the walls of the vacuum vessel are subject to a high particle flux which causes absorbed gases and wall material to be ejected into the plasma. This recycling of plasma fuel, which occurs primarily on the inner graphite limiter, plays an important role in plasma performance. For example, the highest fusion power occurred in the supershot regime which is characterized by a peaked density profile requiring low particle influx from the edge. Despite its importance, a full understanding of the factors influencing recycling is not yet complete.

The operation of a deuterium-tritium (DT) fuel mixture in TFTR has provided an opportunity to study recycling by studying the DT neutron count rate during deuterium-only neutralbeam injected (NBI) discharges. Plasmas fueled by deuterium neutral beams on a deuterium target plasma (DD shots) will contain a few percent of tritium density in the plasma due to wall recycling. Because of the high DT cross section, this small amount of tritium can lead to significant DT neutron count rates. Spectroscopic measurements of the Balmer hydrogen lines can directly measure the tritium influx from the limiter provided the tritium fraction is greater than $2 \%$ of the total hydrogenic influx [1]. In contrast, measurements of the tritium density from the DT neutron count rates are more sensitive (due to the high DT cross section) but less direct, reflecting tritium in the core of the plasma.

In this study, insight into recycling is gained by using this nuclear measurement of tritium density. The factors influencing how the amount of tritium varies from shot to shot are investigated empirically by performing a power-law regression. 


\section{Experiment}

To investigate tritium recycling, it is useful to define an effective tritium density in terms of routine experimental measurements. The total DD and DT neutron rates are given by

$$
\begin{aligned}
& \left.S_{D D}=\int \frac{1}{2} n_{D}^{2}<\sigma v\right\rangle_{D D} d V, \\
& S_{D T}=\int n_{D} n_{T}\langle\sigma V\rangle_{D T} d V,
\end{aligned}
$$

where $n_{D}$ is the deuterium density, $n_{T}$ is the tritium density, the integral is over the volume of the plasma, and $<\sigma v>$ is the mean reaction rate (product of cross section and relative velocity averaged over relative velocities). From Eqs. (1) and (2), the tritium density becomes (neglecting profile effects)

$$
\mathrm{n}_{\mathrm{T}}(0)=\frac{\mathrm{S}_{\mathrm{DT}}}{\mathrm{S}_{\mathrm{DD}}} \mathrm{n}_{\mathrm{e}}(0)\left[\frac{\mathrm{n}_{\mathrm{D}}}{\mathrm{n}_{\mathrm{e}}(0)}\right]\left[\frac{<\sigma \mathrm{V}\rangle_{\mathrm{DD}}}{2<\sigma>_{D T}}\right] .
$$

The ratio $n_{D} / n_{e}(0)$ is the plasma depletion, which will be approximately the inverse of $Z_{\text {eff }}$ (about 2 or 3 for a standard TFTR supershot). Because deuterium and tritium have the same Coulomb barrier, the mean reaction rates have nearly the same functional form and the last factor of Eqn. (3) is approximately constant over a wide range of temperatures [2]. Thus, assuming the last two factors of Eq. (3) are constant, the tritium density in terms of measurable quantities may be defined as

$$
\mathrm{n}_{\mathrm{T}}(0)=\mathrm{C} \frac{\mathrm{S}_{\mathrm{DT}}}{\mathrm{S}_{\mathrm{DD}}} \mathrm{n}_{\mathrm{e}}(0)
$$

where $\mathrm{C}$ is a constant. 
Due to the heuristic nature of this derivation, a comparison of this experimental measure of the tritium density to the tritium density calculated by TRANSP [3][4] was used to test its usefulness. The accuracy of the constant $C$ as defined by Eq. (4) was calculated for 35 supershots using TRANSP. The average value of $C$ in this study was 0.0033 , which was the same to within $10 \%$ for 27 of the shots. Figure 1 shows a comparison between this measurement of the tritium density and TRANSP's calculation during a shot. Regardless of the approximate nature of the derivation, Eq. (4) provides a very useful measurement of the tritium density that is reasonably accurate.

To minimize errors in the above approximation and to reduce variability in recycling behavior, only supershot plasmas are investigated in this report; high-beta plasmas and radiofrequency (RF) wave-driven plasmas are ignored. A typical TFTR supershot plasma in this study has a $5.0 \mathrm{~T}$ toroidal magnetic field, $2.52 \mathrm{~m}$ major radius, and a $0.86 \mathrm{~m}$ minor radius. The neutral beams can supply up to $40 \mathrm{MW}$ of power with sources operating at $95-$ $115 \mathrm{kV}$. To improve the machine conditioning, lithium pellet injection (LPI) is often used. The DT neutron count rates used in this study are measured with surface barrier detectors and the DD count rates are measured with fission and surface barrier detectors [5]. Typical DT and DD neutron rates are shown in Figure 2. The central line-averaged electron density is measured using the Multichannel Far Infrared Interferometer [6].

In this study, the evolution of the tritium density from shot to shot is investigated. The time evolution of the tritium density during a discharge requires consistent measurement times in order for a comparison between shots to be meaningful. The time chosen for this study is $\mathbf{5 0 0}$ milliseconds after the beam ions are turned on. This number is the approximate time after the beams have slowed down, but before a "rollover" in the neutron rate is observed. To minimize noise in the data, the signals are averaged over a 100 millisecond time interval ( $0.45 \mathrm{sec}-0.55 \mathrm{sec}$ after the beams are on). 
A database useful for this type of investigation was created meeting the above requirements. Figure 3 is a collection of all sequences studied with the database. A sequence here is a collection of continuous DD plasma shots all at the same plasma current and similar beam power. There were 13 such sequences in the period from November 1993 to June 1994 comprising 79 DD supershots.

\section{Data Analysis}

Figure 4 illustrates the typical behavior of the evolution of the tritium density from shot to shot. Three features are readily observable: 1) a sequence of DD supershots causes a decay in effective tritium density; 2) a DT supershot causes an increase in the tritium density on the subsequent DD supershot; and 3 ) the overall tritium density in a sequence depends on the amount of tritium already in the limiter surface.

As well as the above three effects, correlations with the tritium density were studied for data often used to measure the wall conditions: carbon influx, visible bremsstrahlung, and hydrogen influx. Also investigated, because they affect the conditioning of the machine, were beam power, plasma current, LPI, disruptions, and carbon blooms.

To quantify the relationships between the tritium density and these parameters, a powerlaw regression was performed on the data. Any parameter that has a statistically significant effect on the influx of tritium from the wall will appear in the resultant scaling equation. The problem that arises in doing this type of analysis is how to quantify the three effects listed above. To overcome this problem, three parameters have been introduced: (1) $\Delta$ is the sequence shot number, defined as the DD shot number minus the DT shot number beginning the sequence, ignoring ohmic and aborted shots. (2) $N_{T}$ is the number of tritium particles injected into the plasma on the DT supershot(s) preceding the sequence. If more than one DT shot precedes the sequence, then the number of tritium particles are summed together. (3) Then "Background" is defined as the tritium density (as defined by Eq. 4) of 
the DD supershot preceding the DT supershot(s) of a given sequence, and provides a rough estimate of the amount of tritium in the limiter surface before the sequence begins.

\section{Scaling Results}

Figure 5 plots the tritium density versus $\Delta$ for a series of ranges of NT times Background. As expected, the tritium density decreases as the number of DD shots increases, and also increases as more tritium is injected and detected (as Background) from the limiter. Quantitatively, the scaling law resulting from a power-law regression is

$$
\mathrm{n}_{\mathrm{T}} \sim \Delta^{-0.46} \mathrm{~N}_{\mathrm{T}}{ }^{0.27} \text { (Background) }^{0.43}
$$

The tritium density versus the scaling law prediction is plotted in Figure 6 to demonstrate the quality of this fit.

In order to quantify the influence of the other parameters, the tritium density is normalized by this scaling equation and plotted versus the other parameters after bin averaging. The plots, which are in Figure 7, show a dependence on the plasma current, albeit a very weak one, but on no other parameter. The introduction of the plasma current into the power-law scaling yields

$$
\mathrm{n}_{\mathrm{T}}=3.21 \times 10^{7} \Delta^{-.45} \mathrm{~N}_{\mathrm{T}}^{0.35} \text { (Background) }{ }^{0.38} \mathrm{I}_{\mathrm{p}}^{-1.4}
$$

where the units are $n_{T}\left[\mathrm{~cm}^{-3}\right]$, NT [particles], Background $\left[\mathrm{cm}^{-3}\right]$, and $\mathrm{l}_{\mathrm{p}}[\mathrm{A}]$. To check the quality of this fit, the tritium density normalized by the new scaling function is again plotted for each parameter in Figure 8. Because no dependencies are shown by any of the parameters, Eq. (6) represents the best fit possible for this data. A plot of the measured tritium density versus the scaling equation, shown in Figure 9, illustrates the good fit. Eq. 6 then is an empirical relation of the tritium density and can be used to predict the tritium density for 
a given DD shot in a sequence. The parameter range over which this regression was performed is shown in the following table.

\begin{tabular}{|l|l||l|l|}
\hline Parameter & \multicolumn{1}{|c|}{ Range } & Parameter & Range \\
\hline$\Delta$ & $1-9$ & Beam Power & $15-30 \mathrm{MW}$ \\
$\mathrm{N} T$ & $9.2 \mathrm{E} 19-3.7 \mathrm{E} 21$ particles & H Influx & $6.7 \mathrm{E} 13-3.0 \mathrm{E} 141 / \mathrm{cm}^{2} \mathrm{~s} / \mathrm{sr}$ \\
Background & $1.0 \mathrm{E} 13-1.5 \mathrm{E} 14 \mathrm{~cm}^{-3}$ & C Influx & $6.7 \mathrm{E} 13-3.0 \mathrm{E} 141 / \mathrm{cm}^{2} \mathrm{~s} / \mathrm{sr}$ \\
Ip & $1.8 \mathrm{E} 6-2.5 \mathrm{E} 6$ Amperes & Vis. Brem. & $4.0 \mathrm{E} 10-2.7 \mathrm{E} 111 / \mathrm{sec}$ \\
\hline
\end{tabular}

Additional regressions have also been carried out in conjunction with this analysis because they might bear on the results. A dependence on the glubal energy confinement time was not seen after performing a similar analysis. Because the tritium density defined uses the electron density, a regression analysis was performed to see what influences that measurement. The results are shown in Figure 10.

\section{v. Discussion}

After a plasma discharge, some of the gas in the wall will escape in a process known as outgassing. The rate $r$ of this outgassing has been described by $r=A t^{-n}$ where $t$ is the time after a discharge, $A$ is a constant, and $n \approx 0.5-1.0$ [7]. Hence, the amount of gas in the vessel after a time $t$ takes the form

$$
n=B-C t 1-n
$$

This dependence is similar to the $\Delta$ dependence found in the scaling relation. Because this simple model does not take into account the deposition or erosion of material on the limiter during a shot, more detailed modeling remains to better understand the cause of the $\Delta$ 
scaling. The $\Delta^{-45}$ result is robust; a similar dependence is seen in $T_{\alpha}[1]$ and neutron activation [8] measurements.

The dependence of NT and Background could possibly indicate that a saturation mechanism is occurring; however, this disagrees with observation of $T_{\alpha}$ measurements that indicate a linear increase in tritium density with fueling. It may be more physically reasonable to sum the two quantities in some manner to represent the tritium available for the sequence, but this is not amenable to a power scaling analysis.

The -1.4 power dependence of the plasma current has a relative error of $22 \%$, which is the greatest relative error of the dependencies. Because there were only 3 different values of the current for in this study, this suggests that the statistical sample may be too small to be meaningful; however, based on the data, there is a relation between the tritium density and the plasma current. This behavior is the least understood and must remain only an observation.

A possible reason for the lack of dependence of hydrogen influx on tritium density is the possibility of different transport mechanisms for tritium and deuterium into the core of the plasma. Because their release from the limiter is a chemical process, it is unlikely that there are differences in their release rates. Also, because of the heuristic derivation of Eq. (4) (especially in the elimination of the integrals), it is possible that systematic differences arise for shots with high edge density. For certain cases, a weak relation is observed between the hydrogen signal and the tritium density can be seen, but systematically it cannot be observed. The largest increase in the hydrogen influx for two consecutive shots is over $100 \%$. The change in the tritium density for these two shots is only $18 \%$, suggesting that the relationship between tritium density and the total hydrogen influx is weak, possibly for the reasons given. In the sequences, the average variation of the $\mathrm{H}_{\alpha}$ signal in two consecutive shots is only $12 \%$ on average. The variation in tritium due to this change 
in the total hydrogen influx, which is somehow reduced, is too small for a correlation to be found.

As illustrated in Figure 11, the hydrogen and carbon influx depend on the power level. Because correlation of the tritium to hydrogen density requires a large variation in the hydrogen influx, a correlation of the tritium density to beam power and carbon influx would also require a large variation in these quantities. The largest difference in beam power is 7.5 MW, which corresponds to the next largest change in hydrogen influx. To see a stronger correlation between tritium density and beam power or carbon influx, larger variations would be needed. We believe that the reason for the lack of large variation in the hydrogen influx is mainly due to a lack of variation in the beam power.

By looking at all of the sequences in Figure 3, no clear conclusion can be reached about the effect of lithium pellet conditioning, disruptions, carbon blooms, and ohmic shots. This conclusion is also supported by Figure 12 which shows the shots in the scaling that follow a disruption and the shots that have lithium conditioning. The lack of a clear dependence of the tritium density on factors that are normally associated with the wall conditions of the machine is somewhat surprising and point to a complicated dependence among these variables. The answer may be that the measurement of the tritium density using neutron data is too indirect to observe the more subtle factors influencing recycling.

The type of analysis done in this study may also miss some of the influences on recycling of tritium because of the time-dependent behavior of the tritium recycling. The DT neutron rate typically peaks anywhere from 400 to 600 milliseconds after the beams are turned on. Some of the fluctuations in the tritium density may be due to factors influencing the time at which the DT neutron rate peaks. Because the scaling law gives such strong correlations with $\Delta, \mathrm{NT}$, and Background, the most important influences have been found; however, a more detailed study of tritium recycling would require a better understanding these most important dependencies. 
In summary, an experimental measure of the tritium density in deuterium-only supershots was defined using the neutron rates and electron density. This experimental measure of the tritium density was used to develop a power-law regression to determine which factors influence the tritium recycling. Possible explanations of the results were suggested although more work is needed to clarify the issues. The scaling law found however, may still be useful as a method of predicting the tritium density in deuterium-only supershot plasmas.

\section{Acknowledgments}

The authors wish to thank the TFTR Team and especially H. Duong, D. Jassby, and L. Johnson for providing and explaining the neutron data. This research was performed under appointment to the Magnetic Fusion Science Fellowship Program administered by Oak Ridge Institute for Science and Education for the U.S. Dept. of Energy.

\section{References}

[1] C.H. Skinner, H. Adler, R.V. Budny, J. Kampershroer, L.C. Johnson, A.T. Ramsey, D.P. Stotler, Nuclear Fusion 35 (1995) 143.

[2] J.D. Strachan, R.E. Chrien, W.W. Heidbrink, J. Vac. Sci. Technol. A1 (1984) 811.

[3] R.V. Budny, Nuclear Fusion 34 (1994) 1247.

[4] R.V. Budny et al. Nuclear Fusion 35 (1995) 544.

[5] J.D. Strachan, et al., Phys. Rev. Lett. 58 (1994) 3526 and references therein.

[6] D.K. Mansfield et al., Applied Optics 15 (1987) 4469.

[7] V. Philips, J. Ehrenberg, J. Vac. Sci. Technol. A, Vac. Surf. Films 11 (1993) 437.

[8] D.K. Owens, private communication. 


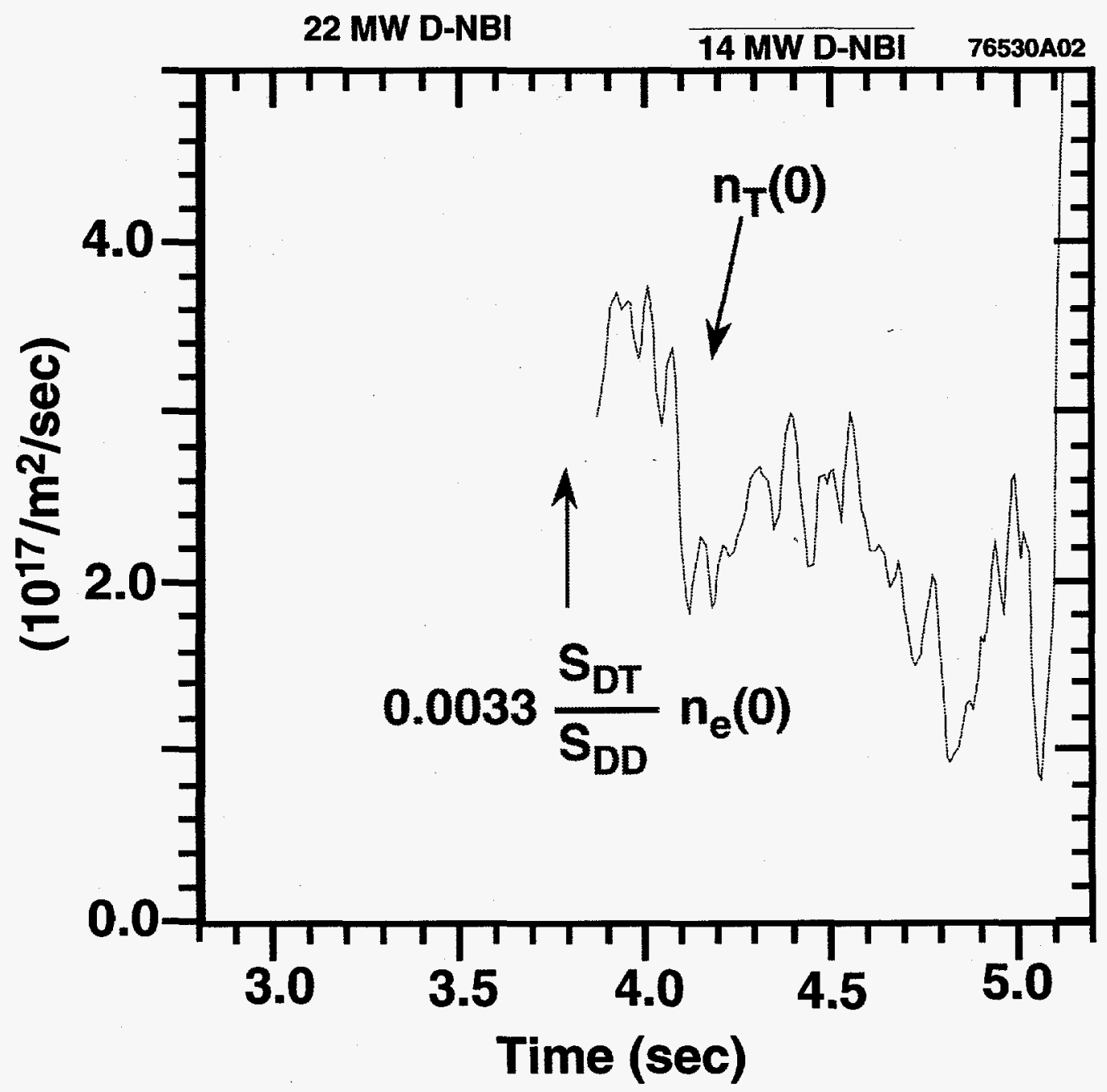

Figure 1 - Comparison between the neutron density as calculated by TRANSP and using Eq. 4 which shows good agreement. The neutron data is unreliable before 2.8 seconds when the beams are turned on. 


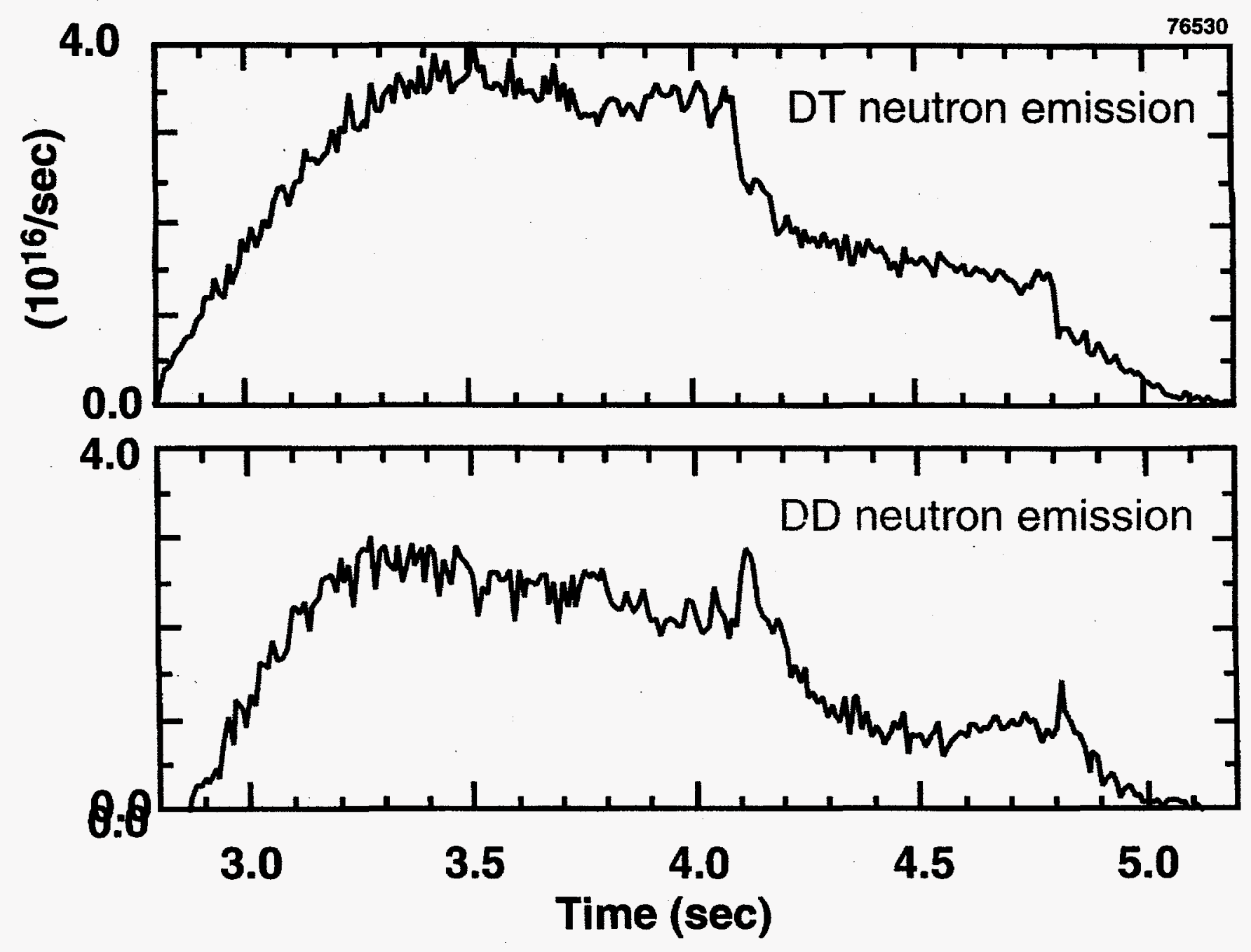

Figure $2-\mathrm{H}_{\alpha}$ and neutron signals for the same shot as in Figure 1. 

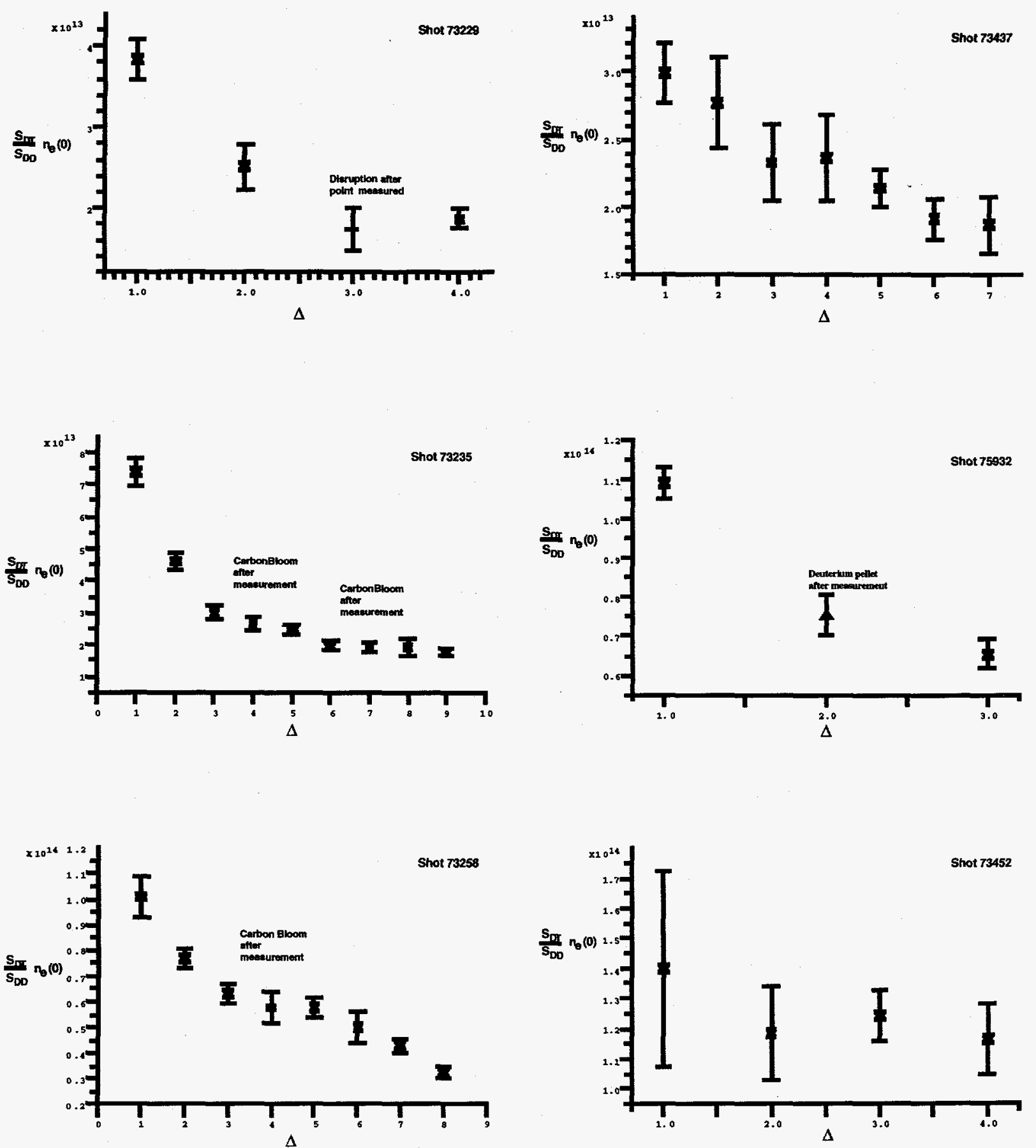

Figure 3 - Sequences of DD shots following a DT shot(s) which is given. Error bars are calculated based on fluctuations in the neutron signals. For the disruption which occurred in the sequence for shot 73229 , the data was not included in the regression analysis because the disruption occurred during the time of data averaging. What is shown was measured before the DT signal saturated. 

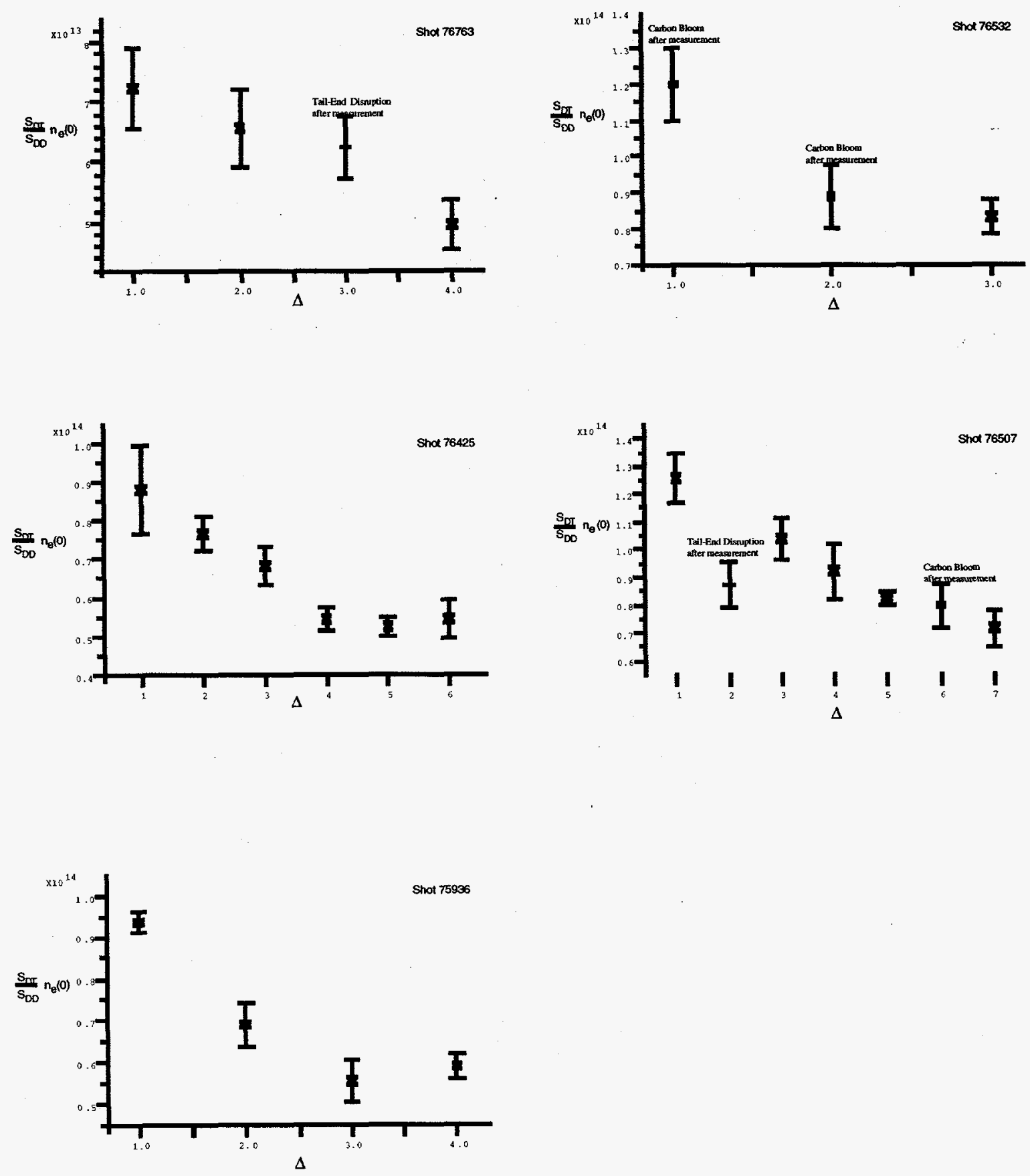

Figure 3 (continued) 


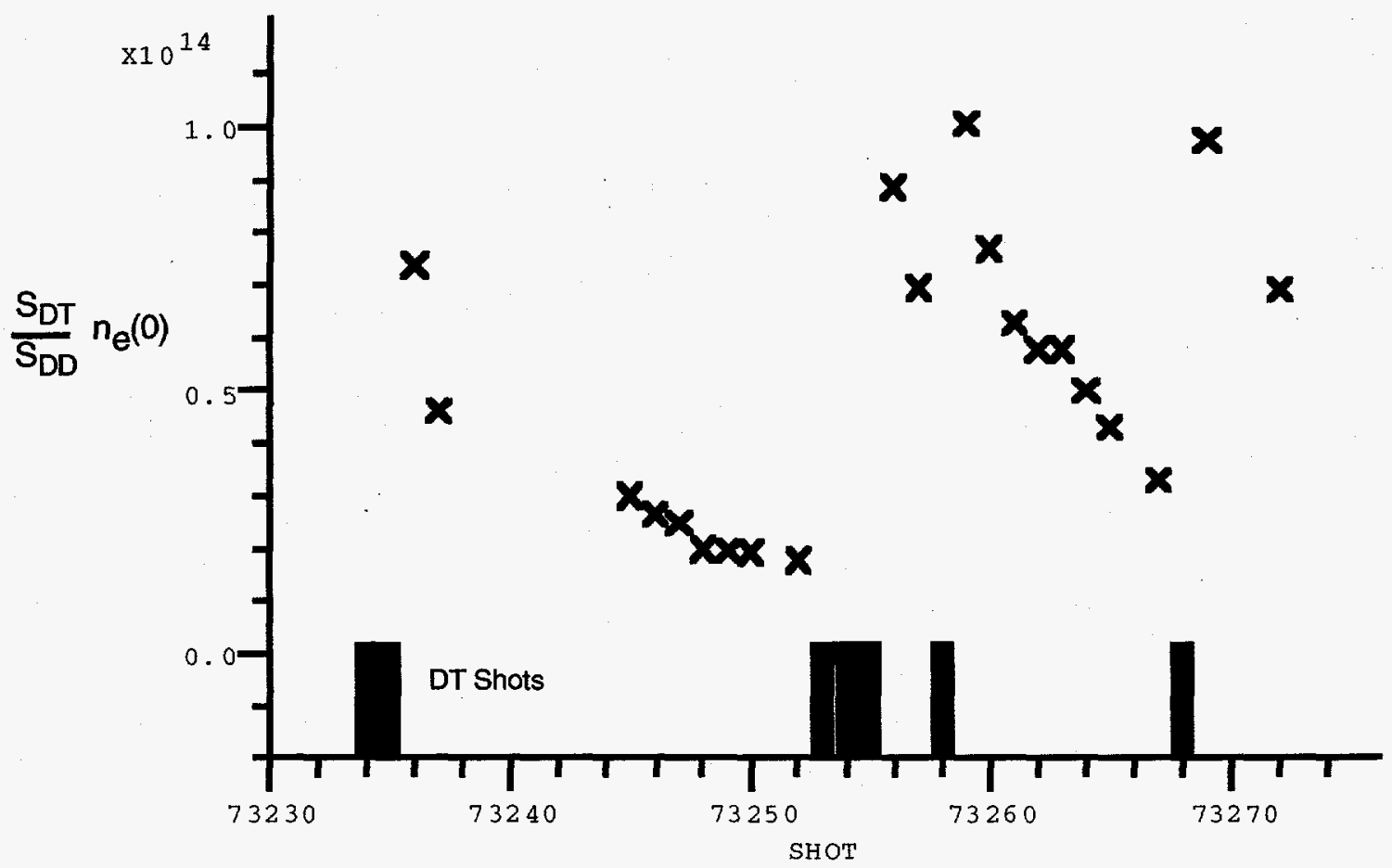

Figure 4 - A typical TFTR run showing the behavior of the tritium density in DD shots which occur after DT shots.

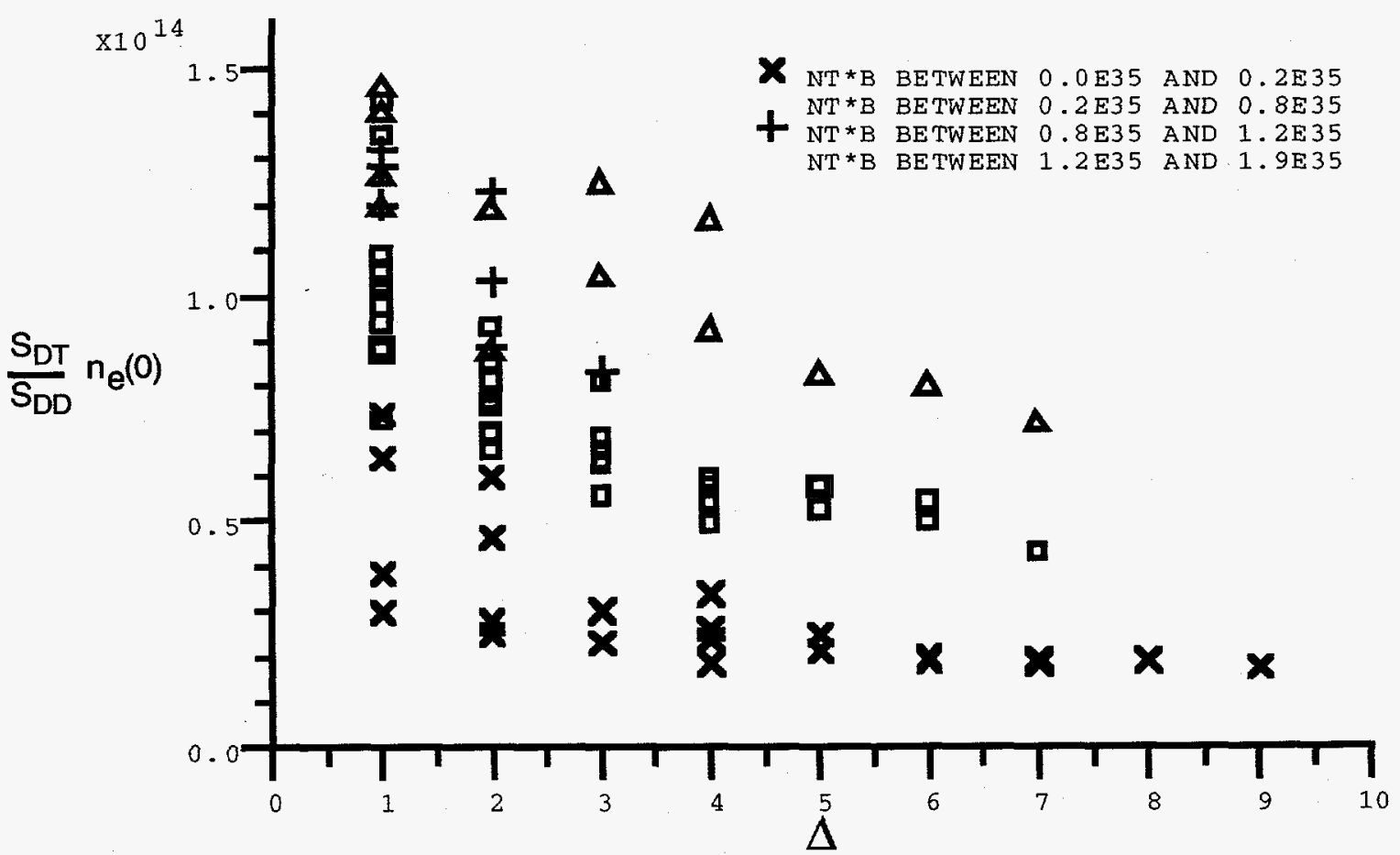

Figure 5 - The tritium density clearly decreases as $\Delta$ increases and increases as $\mathrm{N}_{\mathrm{T}}$ and Background increase. 


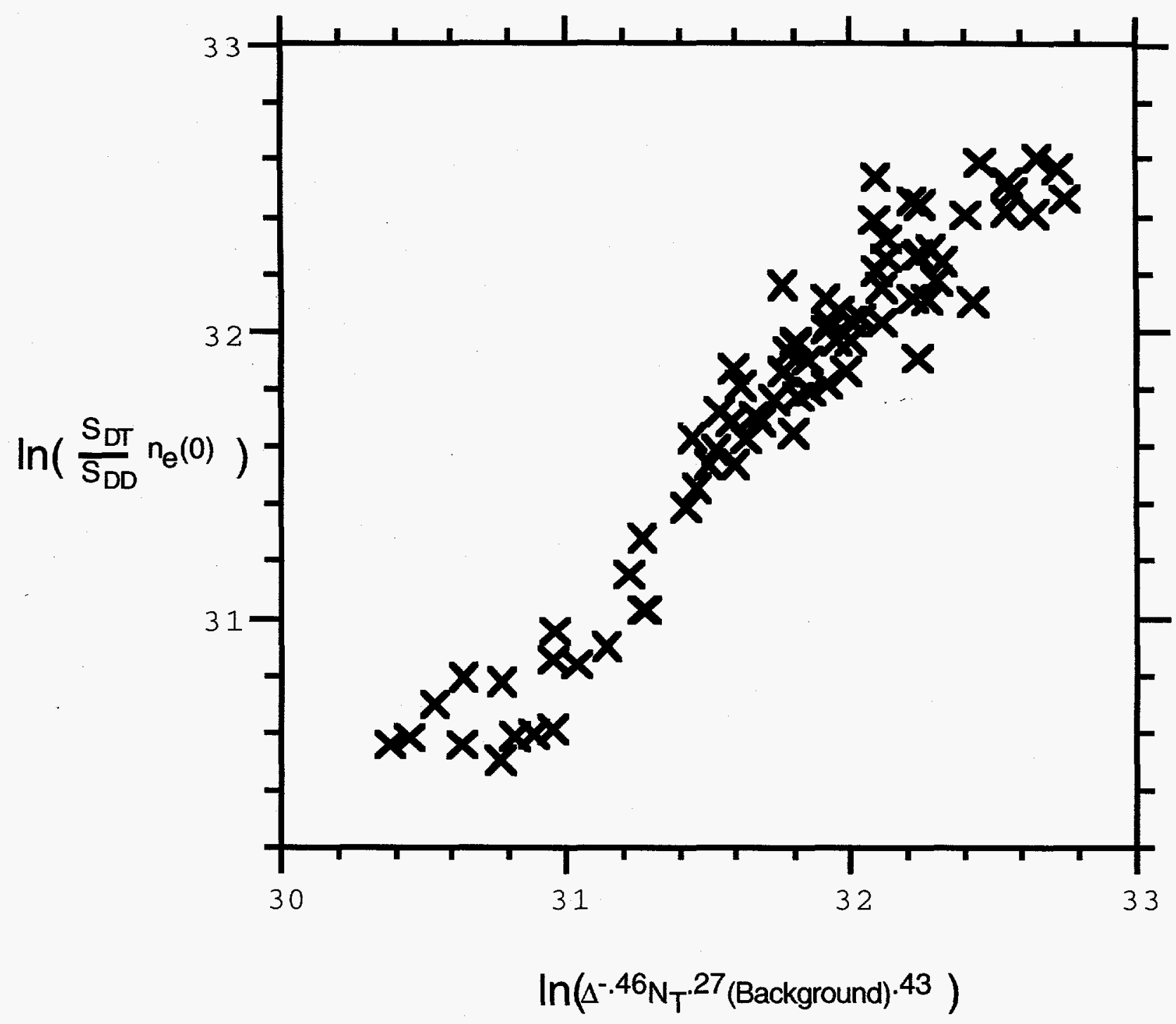

Figure 6 - With a correlation coefficient of 0.923 , the tritium density versus the scaling law of Eq. (5) shows good agreement. A perfect scaling law would show ali data points on a straight line and give a correlation coefficient of 1.00 . 

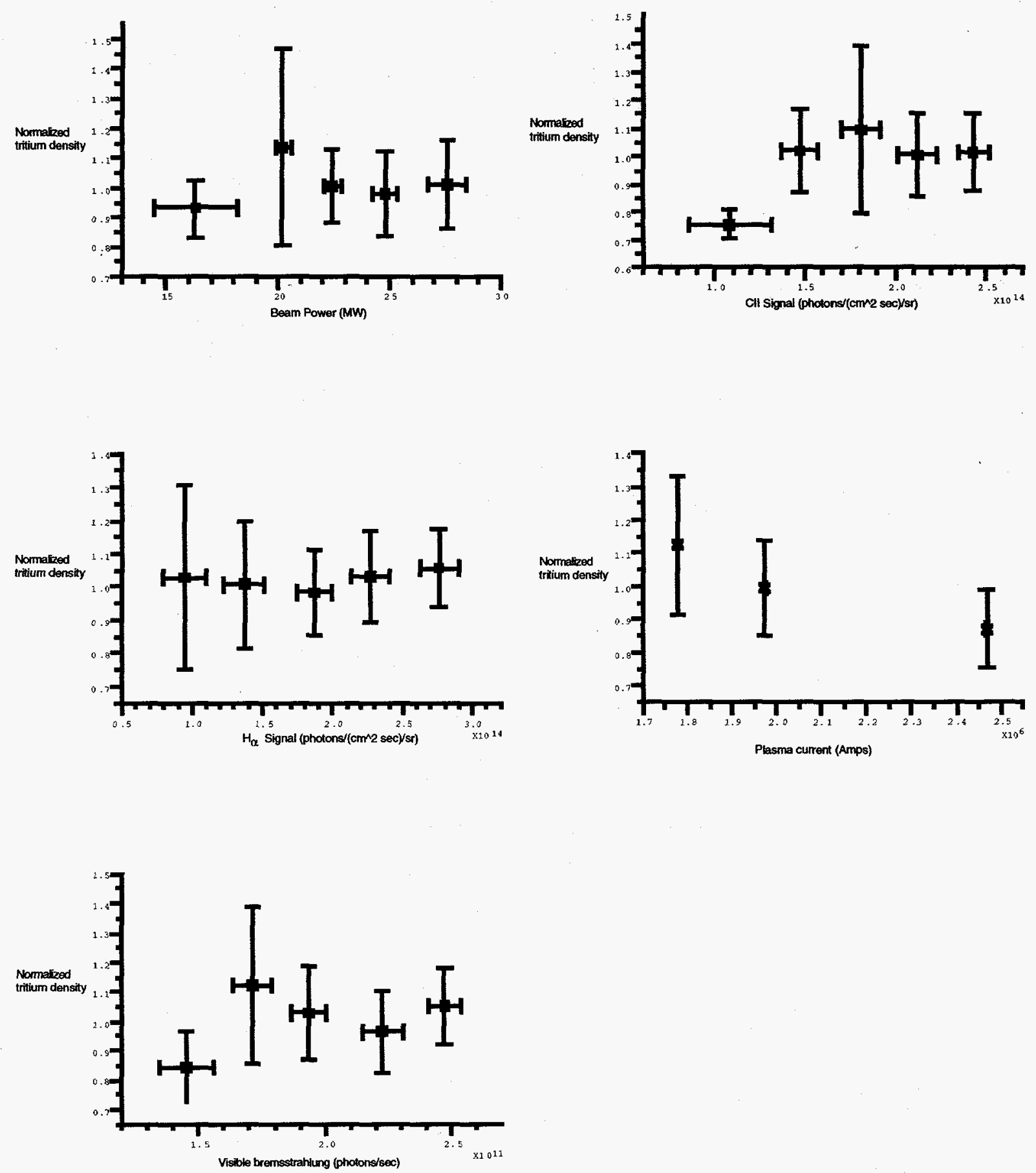

Figure 7 - The bin-averaged tritium density normalized to the scaling law of Equation (5) versus all other parameters which might influence its behavior. Plasma current is the only parameter that shows a dependence. 

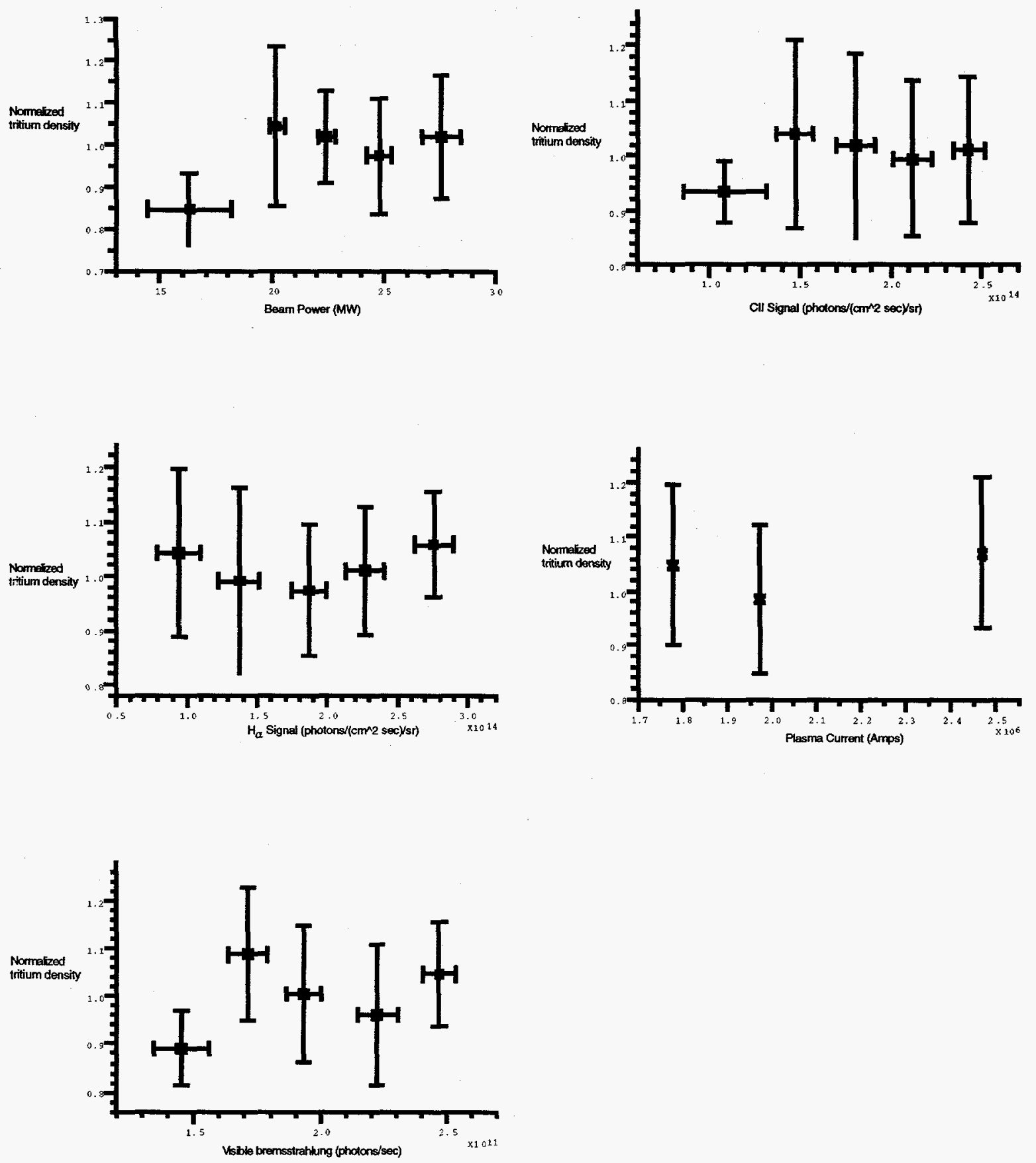

Figure 8 - The bin-averaged tritium density normalized to the scaling law of Eq. (6) versus all other parameters which might influence its behavior. No dependence is seen on any parameter. 


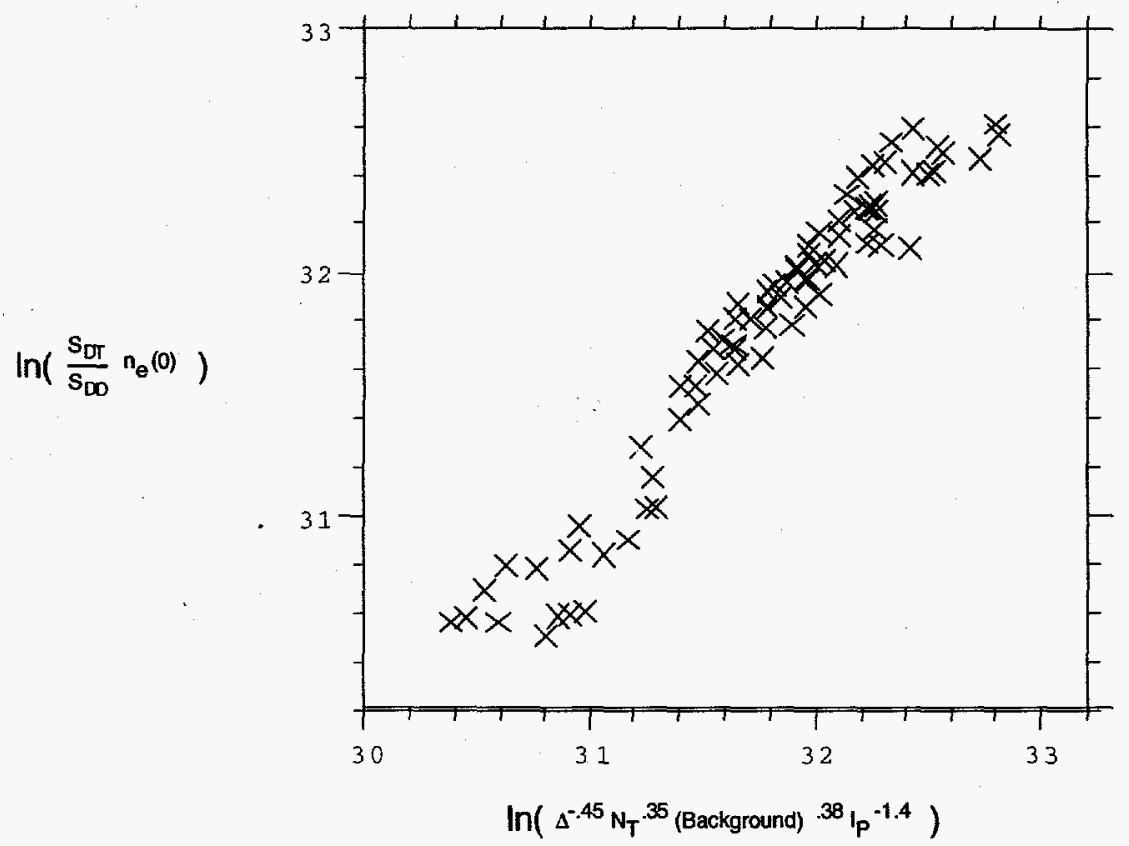

Figure 9 - The tritium density versus the final scaling law including the plasma current shows even better agreement than does Figure 6 . For this fit, the correlation coefficient is 0.939 .

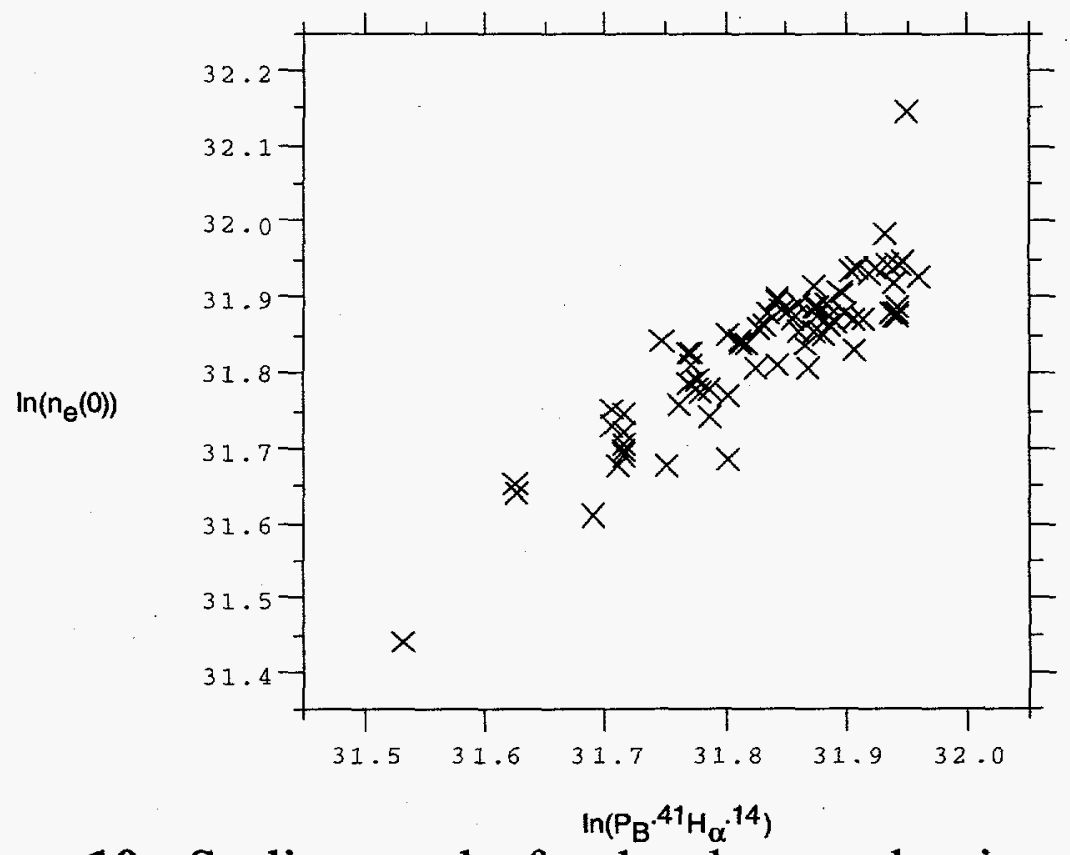

Figure 10 - Scaling results for the electron density covering the same data set as Figure 9. Unlike the tritium density, the electron density scales with beam power, $\mathrm{P}_{\mathrm{B}}$, and the $\mathrm{H}_{\alpha}$ signal. The correlation coefficient is 0.79 . 

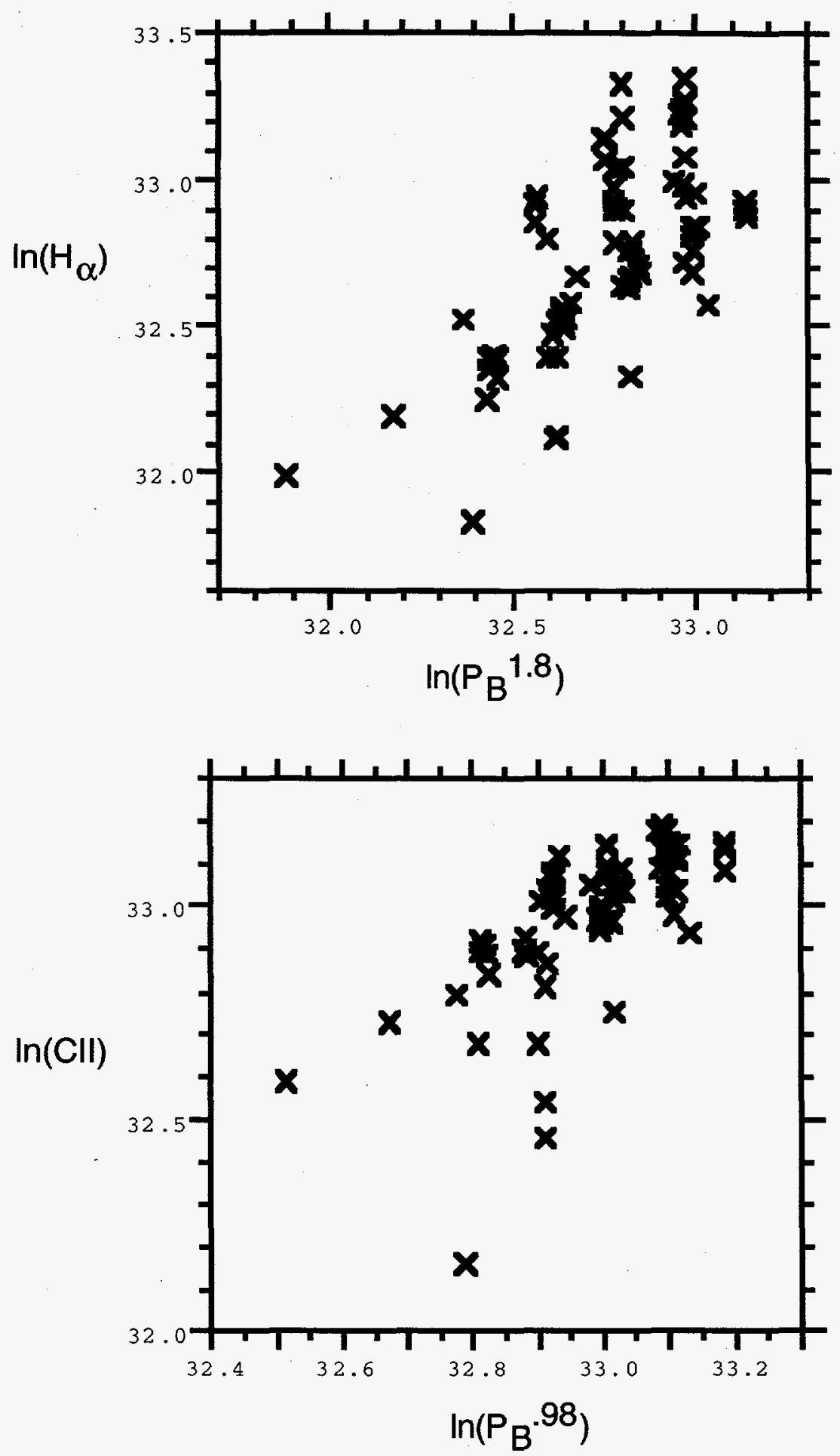

Figure 11 - Figures showing that the $\mathrm{H}_{\alpha}$ and CII signals depend upon neutral beam power 

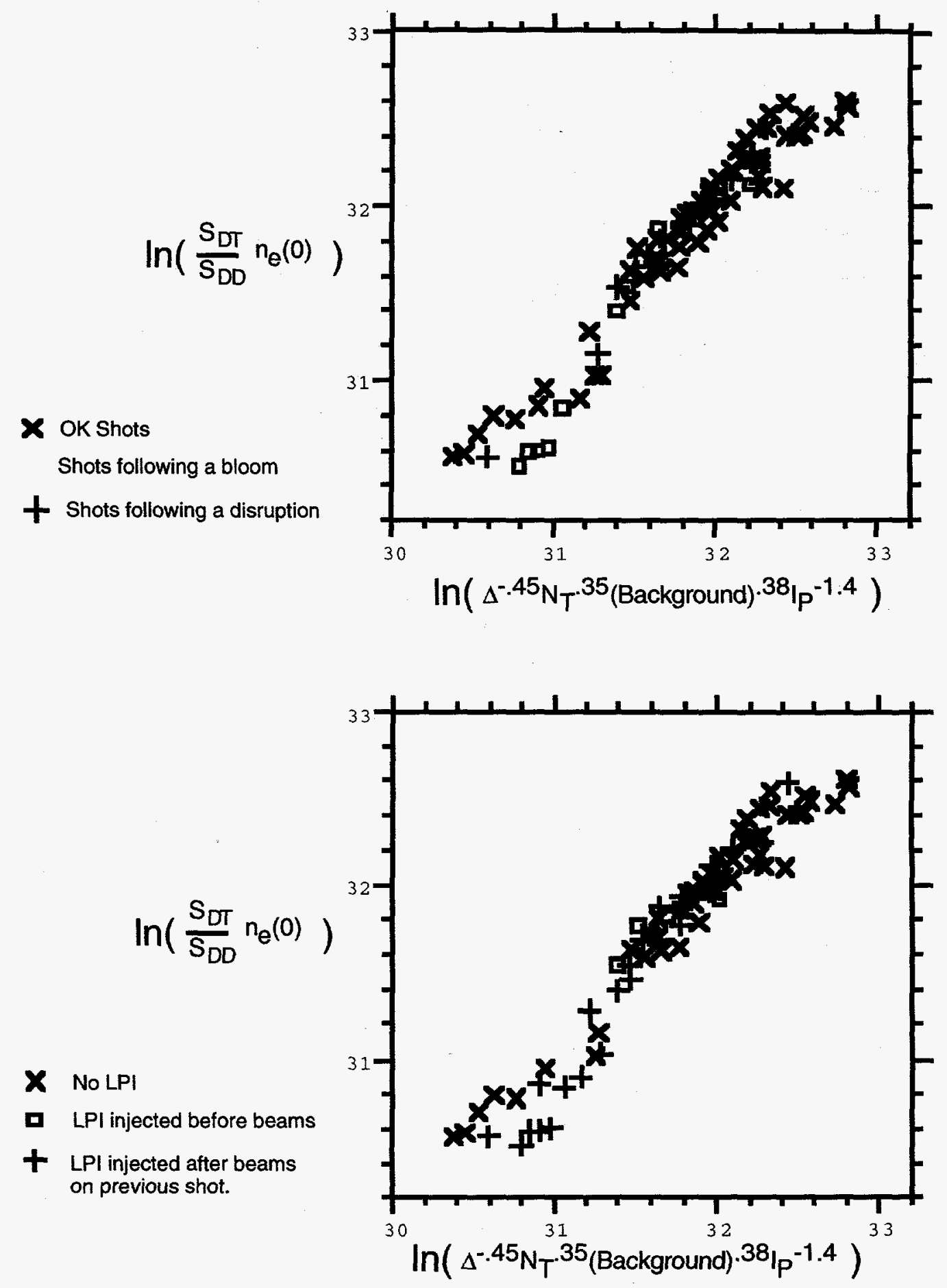

Figure 12 - Carbon blooms, disruptions and lithium pellet injection do not affect the scaling of the tritium density. 
